\title{
Lateralized difference in tympanic membrane temperature: emotion and hemispheric activity
}

\author{
Ruth E. Propper ${ }^{1 *}$ and Tad T. Brunyé 2,3 $^{2}$ \\ ${ }^{1}$ Cerebral Lateralization Laboratory, Psychology Department, Montclair State University, Montclair, NJ, USA \\ ${ }^{2}$ Department of Psychology, Tufts University, Medford, MA, USA \\ ${ }^{3}$ Cognitive Science Team, U.S. Army Natick Soldier Research, Development and Engineering Center, Natick, MA, USA
}

\section{Edited by:}

Marco Hirnstein, University of

Bergen, Norway

\section{Reviewed by:}

Matt Roser, Plymouth University, UK

Ilona Papousek, Karl-Franzens

University of Graz, Austria

*Correspondence:

Ruth E. Propper, Psychology

Department, Montclair State

University, 1 Normal Avenue, 225

Dickson Hall, Montclair, NJ 07043,

USA.

e-mail: propperr@mail.montclair.edu
We review literature examining relationships between tympanic membrane temperature (TMT), affective/motivational orientation, and hemispheric activity. Lateralized differences in TMT might enable real-time monitoring of hemispheric activity in real-world conditions, and could serve as a corroborating marker of mental illnesses associated with specific affective dysregulation. We support the proposal that TMT holds potential for broadly indexing lateralized brain physiology during tasks demanding the processing and representation of emotional and/or motivational states, and for predicting trait-related affective/motivational orientations. The precise nature of the relationship between TMT and brain physiology, however, remains elusive. Indeed the limited extant research has sampled different participant populations and employed largely different procedures and measures, making for seemingly discrepant findings and implications. We propose, however, that many of these discrepancies can be resolved by considering how emotional states map onto motivational systems, and further examining how validated methods for inducing lateralized brain activity might affect TMT.

Keywords: tympanic membrane temperature, motivational orientation, cortical asymmetry, lateralization, mood emotion, hemispheric asymmetry

\section{INTRODUCTION}

The development of techniques allowing examination of brain activity in "real-time" has enabled great strides to be made in the field of behavioral neuroscience generally, and in determining the cortical substrates of emotion and motivational orientation specifically. Electroencephalography and functional imaging have indicated broad differences in hemispheric lateralization of affect/motivation, with the right hemisphere being specialized for negative affect and withdrawal motivation, and the left hemisphere being specialized for positive affect and approach motivation (e.g., Davidson, 2002, 2004; Davidson et al., 1990; Tomarken et al., 1992).

Unfortunately, EEG and functional imaging techniques are expensive, time consuming, limited, or impossible for use in realworld situations, and require specialized knowledge. In order to remove some of these limitations, other methods to assess these relationships have been investigated. One such method is lateralized differences in tympanic membrane temperature (TMT).

Tympanic membrane temperature may reflect hemispheric activity in frontal and temporal lobes (e.g., Schiffer et al., 1999). Increased hemispheric activity is associated with increased propensity to experience that hemisphere's affective/motivational state. For example, individuals with increased right hemisphere activity are more likely to experience negative affect and withdrawal motivation (e.g., Henriques and Davidson, 1991), while those with increased left hemisphere activity are more likely to experience positive affect and approach motivation (e.g., Davidson, 2004). TMT, by reflecting hemispheric activity, may therefore be predictive of emotional and motivational state.
Unfortunately, however, the relationship between TMT and hemispheric activity is by no means straightforward. Although discussion of the physiological mechanisms whereby TMT changes as a function of hemispheric activity is beyond the scope of this mini-review, it should be noted that there are several contending, and contradictory, hypotheses. For simplicity, physiological mechanisms can be grouped into two broad schools of thought, leading to opposite predictions of the relationship between TMT and emotion/motivation; (i) increased TMT on one versus the other side is associated with ipsilaterally increased hemispheric activity (e.g., Boyce et al., 2002; Gunnar and Donzella, 2004) and (ii) increased TMT on one versus the other side is associated with ipsilaterally decreased hemispheric activity (Boyce et al., 1996; Helton, 2010; Helton et al., 2009a). Thus, based on proposed physiological mechanisms alone it is not clear a priori, in any given circumstance, if, for example, increased left TMT is associated with positive emotion [via (i) above] or with negative emotion [via (ii) above].

Examination of the TMT-affect/motivation literature could help elucidate TMT-hemispheric activity relationships, and shed light on the physiological mechanisms that underlie them. Additionally, should a clear predictive relationship between TMT and affect/motivation exist, such a finding would be eminently useful for both research and clinical purposes. From a research perspective, lateralized differences in TMT might enable real-time monitoring of hemispheric activity in real-world conditions. From a clinical perspective, lateralized differences in TMT might serve as a corroborating marker of mental illnesses associated with specific affective dysregulation. 
Thus, the purpose of this mini-review is to (i) determine if lateralized differences in TMT are systematically related to emotion/motivation and (ii) if so, by inference, is increased TMT associated with ipsilaterally increased or with ipsilaterally decreased hemispheric activity.

\section{THE LITERATURE}

A review of the literature revealed a total of 12 articles (including one in press from the authors' laboratories) meeting the following criteria: (i) use of TMT as a dependent measure, (ii) affect or approach/withdrawal behavior as either dependent or independent variables; (iii) analyses allowing determination of affect or approach/withdrawal-TMT relationships; and (iv) humans as participants. Articles were found via a search of PubMed, using various combinations of the key words (and their main root word): "tympanic," "temperature," “emotion," "mood," "lateralization," "asymmetry," and "motivation." Articles were also found via citations listed in articles found using the above methodology.

Some articles, although including TMT and affect/motivation, were eliminated because they failed to meet one of the above criteria (e.g., Schiffer et al., 1999; Parr and Hopkins, 2000; Cherbuin and Brinkman, 2004).

A summary of the results is presented in Table 1.

\section{RESULTS \\ GENERAL}

Twelve articles were produced by five laboratories. One laboratory (Helton and colleagues) produced 42\% (5), while another (Propper and colleagues) produced 25\% (3). An additional laboratory produced 17\% (2; Boyce and colleagues). The remaining two manuscripts came from two different laboratories (Gunnar and Donzella, 2004; Jones et al., 2011). Of the 75\% (9) published after or during 2010, all but one were from the laboratory of either Helton or Propper. These results suggest that investigation of TMT-emotion/motivation is in its infancy, and that while any consistent findings may be promising, they will need to be replicated and extended by other laboratories.

Thirty-three percent (4) examined children 3-9 years of age; the other $67 \%$ (8) investigated TMT in college students. It is not clear if results of young children are generalizable to adults; brain organization, including lateralization of cerebral functions, in addition to structural organization, is subject to developmental changes (e.g., Groen et al., 2012). Because so few TMT studies exist, future research will need to address whether the same processes underlying any TMT-affect/motivational relationships are the same in children and adults.

\section{STUDIES IN CHILDREN}

Three out of four (75\%) studies examining children relied on parental reports of affect/motivational orientation. The fourth (Jones et al., 2011), although investigating TMT in response to stress, did not directly assess affect or motivation, but is included below because it is relevant to the issues discussed here.

The earliest study (Boyce et al., 1996) examined parental reports of behavior and emotional orientation in 8 year old children. Boyce reported that warmer left, relative to right TMT was associated with "decreased ego resilience." Further, examination of their results indicates that increased aggression, externalizing and internalizing behavioral problems, somatization, schizoid behaviors, depression, and social withdrawal were also associated with increased left TMT. Boyce et al. suggested that increased lateralized TMT was related to ipsilaterally decreased hemispheric activity.

Boyce et al. (2002), in an extension and replication, examined TMT-affect/motivation relationships in four cohorts of children ages 4.5-8 years old. In contrast with Boyce et al. (1996), here it was reported that warmer left, relative to right, TMT was associated with positive affect/approach motivation, and warmer right, relative to left TMT was associated with negative affect/withdrawal motivation. Thus, these results suggest the converse of those described above, with warmer left TMT reflecting increased left hemisphere activity, and increased lateralized TMT related to ipsilaterally increased hemispheric activity.

It is not clear why there exists a discrepancy between the two studies; both examined children approximately the same age, and both relied on parental reports of affect/motivational orientation. However, it is notable that Boyce et al. (1996) characterized "aggression" and "problem behaviors" as negative affect/withdrawal motivation. As indicated in Table 2, recent work indicates that "aggression" may be considered an approach motivational state. Additionally, by definition behavioral disinhibition and impulsivity such as that reflected in "externalizing and internalizing behavioral problems," somatization, and schizoid behaviors may be considered left hemisphere, approach behaviors. Though it is not clear why social withdrawal and depression would be associated with increased left TMT, most results reported by Boyce et al. (1996), when considered in this manner, are in agreement with Boyce et al., 2002, such that increased left TMT reflects increased left hemisphere activity.

Gunnar and Donzella (2004), examining parental reports of affect and motivational orientation in 3-5 year olds, reported findings consistent with Boyce et al. (2002); warmer left, relative to right, TMT was associated with positive affect, and warmer right, relative to left TMT was associated with negative affect. Specifically, higher scores on a smiling and laughing scale were associated with warmer left TMT, while higher scores on the sadness scale were associated with warmer right TMT. Interestingly, score on the fear, anger, and shyness scales were not correlated with differential left or right TMT.

Jones et al. (2011) examined TMT in 8 and 9 year old children following a psychosocial stressor. Both left and right TMT decreased following the stressor, and there were no differences between left and right TMT. Birth weight adjusted for placental weight in the children (from previously collected longitudinal data) did correlate with left TMT after stress; children who were smaller babies with bigger placentas had higher left TMT following stress, while larger babies with smaller placentas had warmer right TMT following stress. The authors interpreted their findings as indicating increased right hemisphere activity following stress in children with small sizes and larger placentas.

That is, findings were put into a framework wherein increased lateralized TMT is associated with decreased ipsilateral hemispheric activity. However, it is interesting to note that (i) no measures of stress or mood were examined and (ii) both left and right TMT decreased equally following stress. Additionally, 
Table 1 |Tympanic membrane temperature and affect/motivational orientation articles.

\begin{tabular}{|c|c|c|c|}
\hline Article & Participant age & Task or report & Main findings \\
\hline $\begin{array}{l}\text { Boyce et al. } \\
\text { (2002) }\end{array}$ & 4.5-8 year olds & $\begin{array}{l}\text { Parental report of } \\
\text { affect/behavior }\end{array}$ & $\begin{array}{l}\text { Increased L-R TMT associated with } \\
\text { positive emotions and socially } \\
\text { "competent" behaviors; decreased L-R } \\
\text { TMT associated with negative } \\
\text { emotions and "problem" behaviors }\end{array}$ \\
\hline $\begin{array}{l}\text { Boyce et al. } \\
\text { (1996) }\end{array}$ & 8 year olds & $\begin{array}{l}\text { Parental report of } \\
\text { affect/behavior }\end{array}$ & $\begin{array}{l}\text { Increased L-R TMT associated with } \\
\text { increased aggression, decreased ego } \\
\text { resilience, depression, externalizing, } \\
\text { and internalizing behavior problems, } \\
\text { schizoid behaviors, social withdrawal, } \\
\text { and somatization }\end{array}$ \\
\hline $\begin{array}{l}\text { Gunnar and } \\
\text { Donzella } \\
\text { (2004) }\end{array}$ & $3-5$ year olds & $\begin{array}{l}\text { Parental report of } \\
\text { affect/behavior }\end{array}$ & $\begin{array}{l}\text { Increased L-R TMT associated with } \\
\text { increased smiling and laugher, } \\
\text { decreased L-R TMT associated with } \\
\text { increased sadness }\end{array}$ \\
\hline $\begin{array}{l}\text { Helton } \\
(2010)\end{array}$ & $\begin{array}{l}19.97 \text { Mean } \\
\text { age (under- } \\
\text { graduates) }\end{array}$ & $\begin{array}{l}\text { Impulsivity assessed via } \\
\text { performance on Go-No-Go } \\
\text { tasks }\end{array}$ & $\begin{array}{l}\text { Warmer LTMT associated with } \\
\text { increased errors of commission and } \\
\text { faster reaction time }\end{array}$ \\
\hline
\end{tabular}

\section{Summary}

Warmer LTMT associated with positive/approach emotions; warmer R TMT associated with negative emotions

Warmer LTMT associated with negative/withdrawal emotions

Warmer LTMT associated with positive/approach emotions; warmer R TMT associated with negative emotions

Warmer LTMT associated with increased impulsivity/approach motivation; Increased R TMT associated with more "cautious" behavior

Placed in framework of "threat assessment"; interpretation not

\section{clear \\ TMT when measured by male}

female investigator; Similar $R$ and investigator

Increased R TMT associated with sustained attention to global stimuli performance on local and global sustained attention tasks

Helton et al. 20 Mean age No affect report following

(2009b) (undergraduates) possible mood induction, attention assessed via performance on the sustained attention to response task

\begin{tabular}{|c|c|c|}
\hline $\begin{array}{l}\text { Helton and } \\
\text { Maginnity } \\
\text { (2012) }\end{array}$ & $\begin{array}{l}21 \text { Mean age } \\
\text { (undergradu- } \\
\text { ates) }\end{array}$ & $\begin{array}{l}\text { Self-report of } \\
\text { attention/inattention }\end{array}$ \\
\hline $\begin{array}{l}\text { Jones et al. } \\
\text { (2011) }\end{array}$ & 7-10 year olds & $\begin{array}{l}\text { No affect report following } \\
\text { stress induction }\end{array}$ \\
\hline $\begin{array}{l}\text { Propper } \\
\text { et al. (2010) }\end{array}$ & $\begin{array}{l}19.66 \text { Mean } \\
\text { age (under- } \\
\text { graduates) }\end{array}$ & $\begin{array}{l}\text { Self-report of affect at } \\
\text { baseline }\end{array}$ \\
\hline $\begin{array}{l}\text { Propper } \\
\text { et al. (in } \\
\text { press) }\end{array}$ & $\begin{array}{l}\text { Undergraduates } \\
\text { (age not } \\
\text { reported) }\end{array}$ & $\begin{array}{l}\text { Self-report of affect following } \\
\text { mood induction }\end{array}$ \\
\hline
\end{tabular}

Increased R-L TMT associated with decreased inattention

Low fetal-maternal "health" at birth associated with increased L-R TMT following stress

No association between R-LTMT and any emotion; Ar-ITMT associated with increased anger/hostility

Baseline: increased R TMT associated with increased positive affect; post-manipulation: increased R TMT associated with increased negative affect; increased LTMT associated with positive affect
Warmer RTMT associated with global attention; interpreted as cognitive fatigue-related decreased $\mathrm{R}$ hemisphere activity

Warmer RTMT associated with increased attentiveness

Warmer RTMT associated with increased attentiveness

Warmer L TMT associated with negative emotions

Greater absolute difference between $L$ and RTMT associated with anger/hostility

Greater absolute difference between $L$ and RTMT associated with anger/hostility; other results not clear 
Table 1 | Continued

\begin{tabular}{|c|c|c|c|c|}
\hline Article & Participant age & Task or report & Main findings & Summary \\
\hline $\begin{array}{l}\text { Propper } \\
\text { et al. (2011) }\end{array}$ & $\begin{array}{l}\text { Range of } \\
19-21 \text { years } \\
\text { per group } \\
\text { (undergradu- } \\
\text { ates) }\end{array}$ & $\begin{array}{l}\text { Self-report of affect following } \\
\text { sustained unilateral visual } \\
\text { stimulation }\end{array}$ & $\begin{array}{l}\text { Increased Ar-ITMT associated with } \\
\text { increased anger/hostility, increased R-L } \\
\text { TMT associated with increased anger }\end{array}$ & $\begin{array}{l}\text { Greater absolute difference } \\
\text { between L and R TMT associated } \\
\text { with anger/hostility; warmer R TMT } \\
\text { associated with negative emotions }\end{array}$ \\
\hline
\end{tabular}

Table 2 | Affective valence $\times$ motivational state.

\begin{tabular}{llll}
\hline & \multicolumn{2}{c}{ Motivational state } \\
\cline { 3 - 3 } & & Approach & Avoidance \\
\hline Affective valence & Positive & Enthusiasm & - \\
& & Amusement & \\
& Negative & Desire/pleasure & \\
& Anger & Anxiety \\
& Frustration & Fear \\
& Aggression & Disgust \\
\hline
\end{tabular}

individual differences in birth weight-placental size may be associated with individual differences in any number of other areas, including structural and functional cerebral lateralization, or in overall subjective response to "stress." If so, then the results indicate a relationship such that increased lateralized TMT is associated with ipsilaterally increased hemispheric activity.

The TMT-affect/motivation literature in children offers some limited evidence that (i) warmer left TMT is associated with positive affect/approach motivational states and that warmer right TMT is associated with negative affect/withdrawal motivational states; and (ii) a relationship between lateralized TMT and affect/motivation such that increased lateralized TMT is associated with increased ipsilateral hemispheric activity.

\section{STUDIES IN ADULTS}

The 67\% (8) studies examining TMT-affect/motivation in adults come from two laboratories, Helton and colleagues and Propper and colleagues. All were published in 2010 or later, and all examined college students.

Helton et al. (2009a), Helton and Maginnity (2012), and Helton et al. (2009b) examined adult college students, using self-reported (Helton and Maginnity, 2012) and performance measures (Helton et al., 2009a; Helton et al., 2009b) of attention. One interpretation of focused or sustained attention is that it represents a state of approach motivation (Gable and Harmon-Jones, 2008). In all three studies, warmer right TMT was associated with increased attention. Helton et al. (2009a), investigated sustained attention in a paradigm examining local and global processing. They reported increased right TMT to be associated with increased sustained attention; they interpreted their findings as indicative of right hemisphere fatigue, and of decreased right hemisphere activity. Helton et al. (2009b) reported increased right TMT following exposure to negative pictures, and additionally following performance of a sustained attention to response task, considered as a test of sustained attention. Again, results were interpreted as indicating warmer TMT being associated with decreased ipsilateral hemispheric activity. Finally, Helton and Maginnity (2012) reported that decreased self-reported symptoms of inattention in college students were associated with increased right TMT.

Although Helton et al. (2009a) and Helton et al. (2009b) suggest that increased right TMT is associated with decreased right hemisphere activity, alternate explanations exist. First, clear differences in attentional capacities per se are known to exist between the hemispheres. Specifically, the right hemisphere is known to have a much larger role in attending generally, relative to the left hemisphere (Ramachandran and Blakeslee, 1999). Additionally, the right hemisphere is known to be particularly involved in vigilance (Warm et al., 2009). Thus, when considered from a perspective other than emotion/motivation, the above results are consistent with the notion that increased TMT on one versus the other side reflects ipsilaterally increased hemispheric activity. Similarly, the increased right TMT following exposure to negative pictorial stimuli supports this interpretation, given the right hemisphere's known role in negative affect/withdrawal motivation, and the results reported in studies of children's TMT.

Helton (2010) examined college students, using behavioral measures of impulsivity/approach motivation in an experimental design. In a Go-No-Go task, increased errors of commission, and faster reaction times, were associated with increased left TMT. Findings were interpreted such that increased impulsivity and approach motivation were associated with increased left TMT, and that increased lateralized TMT was indicative of increased ipsilateral hemispheric activity.

Helton and Carter (2011) reported effects of experimenter gender on lateralized TMT, finding warmer left, relative to right TMT when participants were tested by a female investigator. They interpreted their findings as resulting from "threat appraisal," with decreased threat detected with female researchers. Although only minimally discussed, it was suggested that warmer TMT is associated with ipsilaterally decreased hemispheric activity; in this case then, decreased threat is associated with increased right hemisphere activity. However, it is equally plausible that the presence of a female investigator increased approach motivation (relative to a male investigator), and that therefore increased left TMT is associated with ipsilaterally increased hemispheric activity.

Work from the authors' laboratories (Propper et al., 2010, 2011, in press) examined self-reported affect at baseline and following experimental manipulations. Propper et al. (2010) 
examining baseline TMT-affect relationships reported that greater difference between the left and right TMT was associated with increased anger. No other TMT-affect relationships attained significance.

Propper et al. (2011), manipulated hemispheric activity via sustained unilateral gaze to examine effects on TMT. Increased left TMT was associated with increased anger collapsing across all conditions, as was the absolute difference between the left and right TMT. Though interpreted as indicating an association between negative affect (anger) and increased right hemisphere activity (that is, ipsilaterally decreased hemispheric activity), given research indicating that anger is an approach motivational state (Harmon-Jones and Allen, 1998), the findings are at least equally likely to suggest that increased lateralized TMT is associated with increased ipsilateral hemispheric activity. Propper et al. (in press), examined TMT following mood induction. Findings again indicated that increased difference between the left and right TMT, regardless of the direction of that difference, was associated with increased anger. Additionally, tentative evidence was presented suggesting pre versus post-task differences in the relationship between TMT and affect/motivational state, such that pre-task lateralized TMT is associated with ipsilaterally decreased hemispheric activity, but post-task with ipsilaterally increased hemispheric activity. However, the pre-task findings occurred only in men, with few participants, and in only one mood (happiness/sadness) condition. In contrast, post-task associations occurred for both calm/anxious mood and for happiness/sadness, such that lateralized TMT was associated with ipsilaterally increased hemispheric activity.

\section{SUMMARY}

The present review supports the proposal that TMT holds potential for broadly indexing lateralized brain physiology during tasks demanding the processing and representation of emotional and/or motivational states, and for predicting trait-related affective/motivational orientations. The precise nature of the relationship between TMT and brain physiology, however, remains elusive. Indeed the limited extant research has sampled different participant populations and employed largely different procedures and measures, making for seemingly discrepant findings and implications. For example, methodologically, some of the studies reviewed here examined TMT as an indicator of stable, between-subjects individual differences in affective/motivational orientation (e.g.: Boyce et al., 1996; Boyce et al., 2002; Propper et al., 2010; Helton and Maginnity, 2012), while others considered TMT as indicating within-subjects variation in affective/motivational state (e.g., Helton et al., 2009a,b; Propper et al., 2011, in press). Still others examined TMT as reflecting trait differences in response to state manipulations involving stress (e.g., Jones et al., 2011). Interestingly, it has been proposed that within- versus between-subjects examinations may influence TMT-hemispheric relationships, with increased TMT being associated with (i) decreased ipsilateral hemispheric activity in withinsubjects designs and (ii) increased ipsilateral hemispheric activity in between-subjects designs. (e.g., Cherbuin and Brinkman, 2007; Helton, 2010; Propper et al., in press). However, the literature reviewed here reveals no clear pattern for differences in lateralized TMT as a function of consideration of state versus trait variables, and we leave it to others to investigate this suggestion further empirically.

We do propose, however, that many of the discrepancies in the TMT literature can be resolved by considering how emotional states map onto motivational systems (i.e., Table 2), and further examining how validated methods for inducing lateralized brain activity might affect TMT.

Traditional theories attempting to define emotional experience considered emotions as modular entities (e.g., Izard, 1991; Ekman, 1992), or sought to describe their underlying dimensions of valence and arousal (Lang et al., 1992; Russell, 2003). More recent work has demonstrated utility in defining not only the modularity of basic emotions or their underlying valence and arousal, but also considering the nature of events that elicit and reinforce emotions. The events associated with particular emotions carry underlying states that motivate certain types of behavior (Frijda, 1986; Davidson, 1998). In general, these motivational states are associated with approach or avoidance motives, both considered core elements in the organization of human behavior (Carver and Harmon-Jones, 2009). Critically, approach and avoidance motivations are reliably associated with distinct neural substrates thought to reside in the left and right brain hemispheres, respectively (Davidson, 1998; Pizzagalli et al., 2005). Thus, while affective states such as anger and fear are decidedly negative in valence and high arousal, they are associated with opposing motivational systems; anger promotes approach-related motives whereas fear promotes avoidance. With these distinctions in mind, we have suggested that many seemingly discrepant results can be at least partially resolved by considering how individual emotions map onto underlying motivational states.

More difficult to reconcile, however, are mixed reports detailing the directionality of any relationships between TMT and hemispheric activity. Several studies suggest that increased TMT is associated with decreased ipsilateral hemispheric activity (e.g., Helton et al., 2009a,b; Helton and Maginnity, 2012); others suggest the opposite (Helton and Carter, 2011). Still others suggest that hemispheric differences in TMT, regardless of direction, might reliably indicate the presence of an approach-oriented emotion such as anger (Propper et al., 2010, in press). Future research might attempt to replicate this latter effect with emotional states characterized by opposing motivations, such as fear. Individual differences in lateralization of emotion may have contributed to inconsistent relationships; research might control for these individual differences by ensuring that all participants are strongly right-handed.

We propose at least three types of continuing research that might prove successful in further detailing the relationship between TMT and emotional and motivational states. First, we propose that if TMT can be used to reliably index hemispheric activity, then actively promoting neural activity in specific hemispheres, such as via sustained unilateral gaze or trans-cranial direct current stimulation (tDCS), should elicit reliable differences in TMT. Some recent work in our laboratories suggests that this might be the case (Propper et al., 2011). Low-current 
brain stimulation techniques, such as tDCS might also prove useful in temporarily increasing neural activity in one or both brain hemispheres. Second, we propose functional neuroimaging techniques such as EEG should be used to assess potential relationships between TMT, affect, and process-specific frequency bands. As reviewed above, we are aware of only one such study (Schiffer et al., 1999). Third, we propose value to continuing research examining motivational states associated with particular emotions; for instance, studies examining TMT responses to viewing stimuli reliably associated with appetitive (approach-related) versus disgusting (avoidance-related) motives.

Finally, we'd like to point out that not only may emotional/motivational states be lateralized, but so too are many cognitive processes (e.g., Hellige, 2001), offering the possibility that TMT may also reflect cognition. In fact, research suggests that TMT may reflect lateralized cognitive performance in some domains. For example, performance on a visuo-spatial task demonstrated decreased TMT, while performance on a verbal task resulted in decreased left TMT (Cherbuin and Brinkman, 2004).

\section{REFERENCES}

Boyce, W. T., Essex, M. J., Alkon, A., Smider, N. A., Pickrell, T., and Kagan, J. (2002). Temperment, tympanum, and temperature: four provisional studies of the biobehavioral correlates of tympanic membrane temperature asymmetries. Child Dev. 73, 718-733.

Boyce, W. T., Higley, D., Jemerin, J. J., Champoux, M., and Suomi, S. J. (1996). Tympanic temperature asymmetry and stress behavior in Rhesus Macaques and children. Arch. Pediatr. Adolesc. Med. 150, 518-523.

Carver, C. S., and Harmon-Jones, E. (2009). Anger is an approachrelated affect: evidence and implications. Psychol. Bull. 135, 183-204.

Cherbuin, N., and Brinkman, C. (2004). Cognition is cool: can hemispheric activation be assessed by tympanic membrane thermometry? Brain Cogn. 54, 228-231.

Cherbuin, N., and Brinkman, C. (2007). Sensitivity of functional tympanic membrane thermomentry (fTMT) as an index of hemispheric activation in cognition. Laterality 12, 239-261.

Davidson, R. J. (1998). Affective style and affective disorders: perspectives from affective neuroscience. Cogn. Emot. 12, 307-320.

Davidson, R. J. (2002). Anxiety and affective style: role of prefrontal cortex and amygdala. Biol. Psychiatry 51, 68-80.

Davidson, R. J. (2004). Well-being and affective style: neural substrates and biobehavioral correlates. Philos.
Trans. R. Soc. Lond. B Biol. Sci. 359, 1395-1411.

Davidson, R. J., Ekman, P., Saron, C. D., Senulis, J. A., and Friesen, W. V. (1990). Approach-withdrawal and cerebral asymmetry: emotional expression and brain physiology I. J. Pers. Soc. Psychol. 58, 330-341.

Ekman, P. (1992). An argument for basic emotions. Cogn. Emot. 6, 169-200.

Frijda, N. O. (1986). The Emotions. Cambridge: Cambridge University Press.

Gable, P. A., and Harmon-Jones, E. (2008). Approach-motivated positive affect reduces breadth of attention. Psychol. Sci. 19, 476-482.

Groen, M. A., Whitehouse, A. J. O., Badcock, N. A., and Bishop, D. V. M. (2012). Does cerebral lateralization develop? A study using functional transcranial Doppler ultrasound assessing lateralization for language production and visuospatial memory. Brain Behav. 2, 256-269.

Gunnar, M. R., and Donzella, B. (2004). Tympanic membrane temperature and emotional dispositions in preschool-aged children: a methodological study. Child Dev. 75, 497-504.

Harmon-Jones, E., and Allen, J. J. B. (1998). Anger and frontal brain activity: EEG asymmetry consistent with approach motivation despite negative affective valence. J. Pers. Soc. Psychol. 74, 1310-1316.

Hellige, J. B. (2001). Hemispheric Asymmetry: What's Right and What's Left. Cambridge, MA: Harvard University Press.

Thus, TMT, via being indicative of hemispheric activity, may also have potential in investigations of lateralized cognitive activity.

\section{CONCLUSION}

Sampling brain activity in "real-time" using portable and noninvasive technologies holds promise for understanding neurophysiologic correlates of real-world experiences. Though very few studies have examined whether TMT might hold value toward this goal, the extant data suggest that TMT is indeed sensitive to manipulations of emotional and/or motivational states. Precisely defining these relationships will be a critical goal for continuing research in this exciting area.

\section{ACKNOWLEDGMENTS}

Parts of this work was supported by U.S. Army contract \#W911QY12-C-0046 to author Ruth E. Propper. The opinions expressed herein are those of the authors and not necessarily of the U.S. Army. The authors thank Sean E. McGraw for locating references and formatting.

Helton, W. S. (2010). The relationship between lateral differences in tympanic membrane temperature and behavioral impulsivity. Brain Cogn 74, 75-78.

Helton, W. S., and Carter, J. R. (2011). The effect of investigator gender on lateral tympanic membrane temperature. Laterality 16, 156-163.

Helton, W. S., Harynen, L., and Schaeffer, D. (2009a). Sustained attention to local and global target features is different: performance and tympanic membrane temperature. Brain Cogn. 71, 9-13.

Helton, W. S., Kern, R. P., and Walker, D. R. (2009b). Tympanic membrane temperature, exposure to emotional stimuli and the sustained attention to response task. J. Clin. Exp. Neuropsychol. 31, 611-616.

Helton, W. S., and Maginnity, M. (2012). Increased attentiveness is associated with hemispheric asymmetry measured with lateral tympanic membrane temperature in humans and dogs. Exp. Brain Res. 219, 321-326.

Henriques, J. B., and Davidson, R. J. (1991). Left frontal hypoactivation in depression. J. Abnorm. Psychol. 100, 535-545.

Izard, C. E. (1991). The Psychology of Emotions. New York: Plenum Press.

Jones, A., Osmond, C., Godfrey, K. M., and Phillips, D. I. (2011). Evidence for developmental programming of cerebral laterality in humans. PLoS ONE 6:e17071. doi:10.1371/journal.pone.0017071

Lang, P. J., Bradley, M. M., and Cuthbert, B. N. (1992). A motivational analysis of emotion: reflex-cortex connections. Psychol. Sci. 3, 44-49.
Parr, L. A., and Hopkins, W. D. (2000). Brain temperature asymmetries and emotional perception in chimpanzees, Pan troglodytes. Physiol. Behav. 71, 363-371.

Pizzagalli, D. A., Sherwood, R. J., Henriques, J. B., and Davidson, R. J. (2005). Frontal brain asymmetry and reward responsiveness: a sourcelocalization study. Psychol. Sci. 10, 805-813.

Propper, R. E., Brunye, T. T., Christman, S. D., and Bologna, J. (2010). Negative emotional valence is associated with non-right-handedness and increased imbalance of hemispheric activation as measured by tympanic membrane temperature. J. Nerv. Ment. Dis. 198, 691-694.

Propper, R. E., Januszewski, A., Brunyé, T. T., and Christman, D. (in press). Tympanic membrane temperature, hemispheric activity, and affect: evidence for a modest relationship. J. Clin. Exp. Neuropsychol.

Propper, R. E., Januszewski, A., Christman, S. D., and Brunye, T. T. (2011). Increased anger is associated with increased hemispheric asymmetry. $J$. Nerv. Ment. Dis. 199, 716-720.

Ramachandran, V. S., and Blakeslee, S. (1999). Phantoms in the Brain: Probing the Mysteries of the Human Mind. New York: William Morrow.

Russell, J. A. (2003). Core affect and the psychological construction of emotion. Psychol. Rev. 110, 145-172.

Schiffer, F., Anderson, C. M., and Teicher, M. H. (1999). Electroencephalogram, bilateral ear temperature, and affect changes induced by lateral visual field stimulation. Compr. Psychiatry 40, 221-225. 
Tomarken, A. J., Davidson, R. J., Conflict of Interest Statement: The Wheeler, R. E., and Doss, R. C. authors declare that the research was (1992). Individual difference in anterior brain asymmetry and fundamental dimensions of emotion. J. Pers. Soc. Psychol. 62, 676-687.

Warm, J. S., Matthews, G., and Parasuraman, R. (2009). Cerebral hemodynamics and vigilance. Mil. Psychol. 21(Suppl. 1), S75-S100. conducted in the absence of any commercial or financial relationships that could be construed as a potential conflict of interest.

Received: 07 December 2012; paper pending published: 23 January 2013; accepted:
13 February 2013; published online: 04 March 2013.

Citation: Propper RE and Brunyé TT (2013) Lateralized difference in tympanic membrane temperature: emotion and hemispheric activity. Front. Psychol. 4:104. doi: 10.3389/fpsyg.2013.00104

This article was submitted to Frontiers in Cognition, a specialty of Frontiers in Psychology.
Copyright (C) 2013 Propper and Brunyé. This is an open-access article distributed under the terms of the Creative Commons Attribution License, which permits use, distribution and reproduction in other forums, provided the original authors and source are credited and subject to any copyright notices concerning any third-party graphics etc. 